\title{
Multilevel Lumbar Stenosis Caused by Large Tophi Involving Both Spinal Canal and Posterior Spinal Elements: A Rare Case Report and Literature Review
}

\author{
Yang Liu ${ }^{\mathrm{a}}$, Xueshi Li ${ }^{\mathrm{a}}$, , Genlong Jiao ${ }^{\mathrm{a}}$, Pan Zhou ${ }^{\mathrm{a}}$, Zhizhong $\mathrm{Li}^{\mathrm{a}}$, b
}

\begin{abstract}
Tophus is a characteristic manifestation of gout entering the chronic phase, which usually deposits in the joints of the extremities, skin mucosa, etc. A gout tophus that involves the spine causing spinal stenosis is rare and it can be misdiagnosed as a spinal tumor preoperatively. We report the case of a 35-year-old man who presented with lumbar stenosis symptoms, and suffered multiple-site gout tophi involvement throughout his body. Radiographic examinations showed that the large tophi infiltrated the posterior elements of the spine and encroached the lumbar spinal canal, resulting in neurologic compression from the second to the fifth lumbar level. Urate could be observed on a dual-energy computed tomography. A posterior-based procedure was performed to eradicate the tophi and stabilize the spine. Finally, urate crystal was confirmed by postoperative pathological examination. Here, the clinical manifestations, radiological, pathological and surgical features for this case are reported.
\end{abstract}

Keywords: Gout; Tophus; Tophi; Lumbar spine; Spinal stenosis

\section{Introduction}

Gout is caused by a metabolic disorder of purine, which is characterized by acute and chronic arthritis, as well as tophus while it enters the chronic phase. The formation of tophi can lead to erosion of articular cartilage/bone and fibrosis of peripheral soft tissue. However, the spine is less eroded by tophi, and involvement of the neurologic system is even rarer. To our knowledge, altogether 152 cases of spinal tophi have been reported in the current literature with less than eight cases hav-

Manuscript submitted November 12, 2019, accepted November 20, 2019

aDepartment of Spinal Surgery, The First Affiliated Hospital of Jinan University, Guangzhou City, Guangdong Province, China

bCorresponding Author: Xueshi Li, The Department of Spinal Surgery, The First Affiliated Hospital of Jinan University, 613 west Huangpu Avenue, Guangzhou 510630, China. Email: xshli777@hotmail.com; Zhizhong Li, The Department of Spinal Surgery, The First Affiliated Hospital of Jinan University, 613 west Huangpu Avenue, Guangzhou 510630, China.

Email: lizhizhongjnu@163.com

doi: https://doi.org/10.14740/jmc3392 ing tophaceous spinal canal compression over more than four lumbar levels [1-4]. Here we report a rare case of giant tophi involving both the spinal canal and posterior spinal elements, resulting in secondary multilevel lumbar stenosis from the second to the fifth lumbar level. By a posterior approach, we successfully performed a decompression and stabilization through the lumbar segments involved. The manifestations of spinal tophi are non-specific, which makes it difficult to distinguish from spinal neoplasm and infection in clinical practice. Therefore, clinicians need to be alert to this disorder, especially for those in institutions without dual-energy computed tomography (DECT) equipment.

\section{Case Report}

A 35-year-old man was admitted to our department because of lower limb weakness, numbness and lower back pain. The lower limb weakness aggravated during the past year with obvious intermittent claudication in the recent 2 months. The strengths of bilateral quadriceps, biceps femoris, tibialis anterior, gastrocnemius and extensor pollicis longus were documented as grade 4 with decreased bilateral knee and ankle reflexes. Diffuse tophi around the four limbs could be observed with partial redness and ulceration (Fig. 1). The visual analogue scale (VAS) score for back pain was 6. The Japanese Orthopedic Association (JOA) and Oswestry disability index (ODI) scores were 14 and $15(30 \%)$, respectively.

The urine and blood uric acid (UA) levels were 933 $\mu \mathrm{mol} / \mathrm{L}$ and $650 \mu \mathrm{mol} / \mathrm{L}$, respectively. The urine and blood creatinine levels were $2,904 \mu \mathrm{mol} / \mathrm{L}$ and $58 \mu \mathrm{mol} / \mathrm{L}$, respectively. The white blood count (WBC), erythrocyte sedimentation rate $(\mathrm{ESR})$ and $\mathrm{C}$-reactive protein $(\mathrm{CRP})$ were $6.36 \times$ $10^{9} / \mathrm{L}, 120 \mathrm{~mm} / \mathrm{h}$ and $13.61 \mathrm{mg} / \mathrm{L}$, respectively. X-rays of the four limbs and spine revealed lumbar (L2-5) and multiple-joint involvement by tophi (Fig. 2). Magnetic resonance imaging (MRI) with gadolinium contrast showed tophaceous change at lumbar 2-5 levels with secondary lumbar stenosis (L2-5) (Fig. 3 ). DECT demonstrated UA deposition at lumbar 2-5 levels and lumbar stenosis (Fig. 4).

Posterior L2-5 tophi excision, spinal decompression and pedicle screw-rod stabilization were performed while the patient was under general anesthesia. The tophi were around 10 $\times 10 \times 5 \mathrm{~cm}$ with pervasive erosion of paraspinous muscles, spinous processes, facet joints, laminae and pedicles. Spinal 

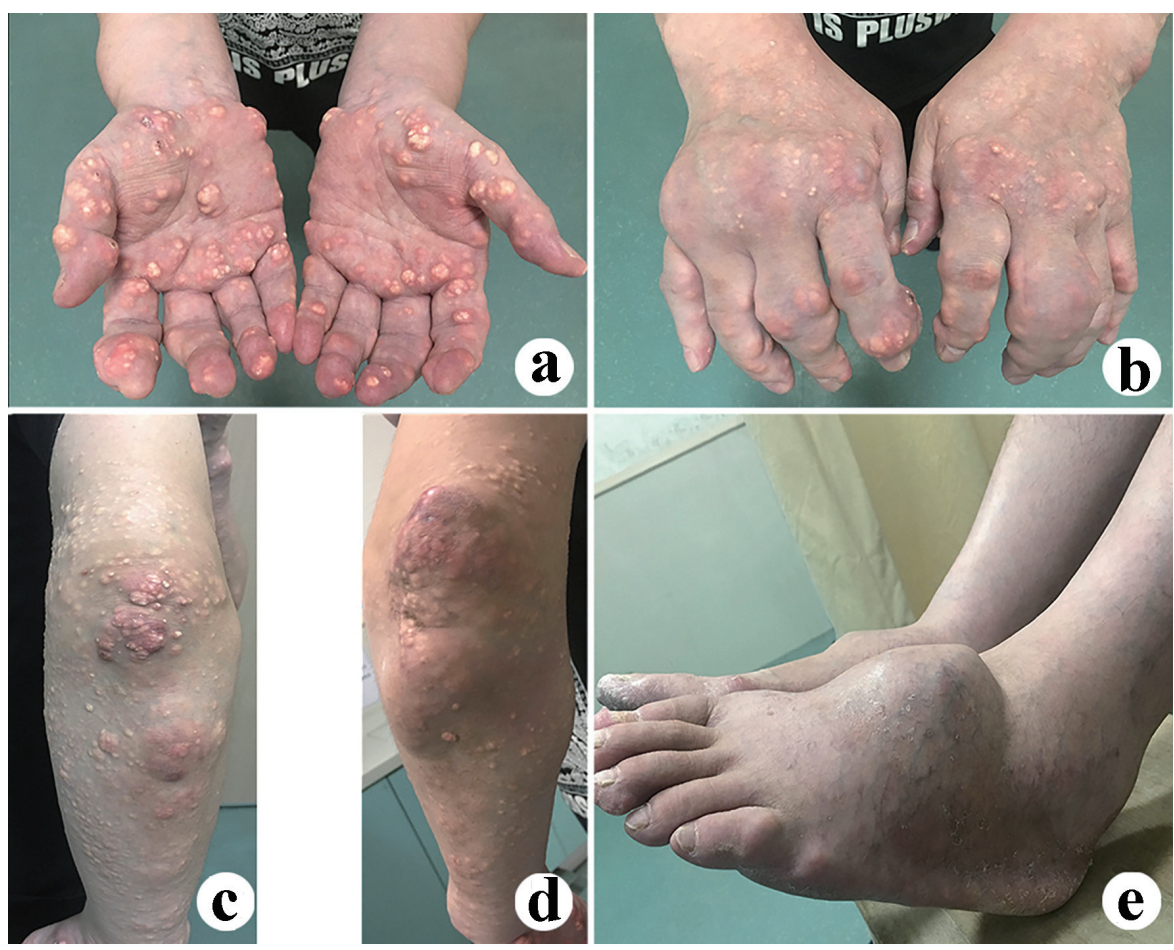

Figure 1. Clinical photos show tophi erosions throughout the patient's four limbs. (a, b, c, d) Upper limbs; (e) Bilateral feet.

decompression was performed across L2-5 central canal and lateral recesses with adequate neurologic decompression. Adhesions were found between the dura and the tophi, which were gently separated under an operative microscope without dural leak. The pedicle screw purchase was sufficient to maintain spinal stability. The patient was ambulating at postopera-
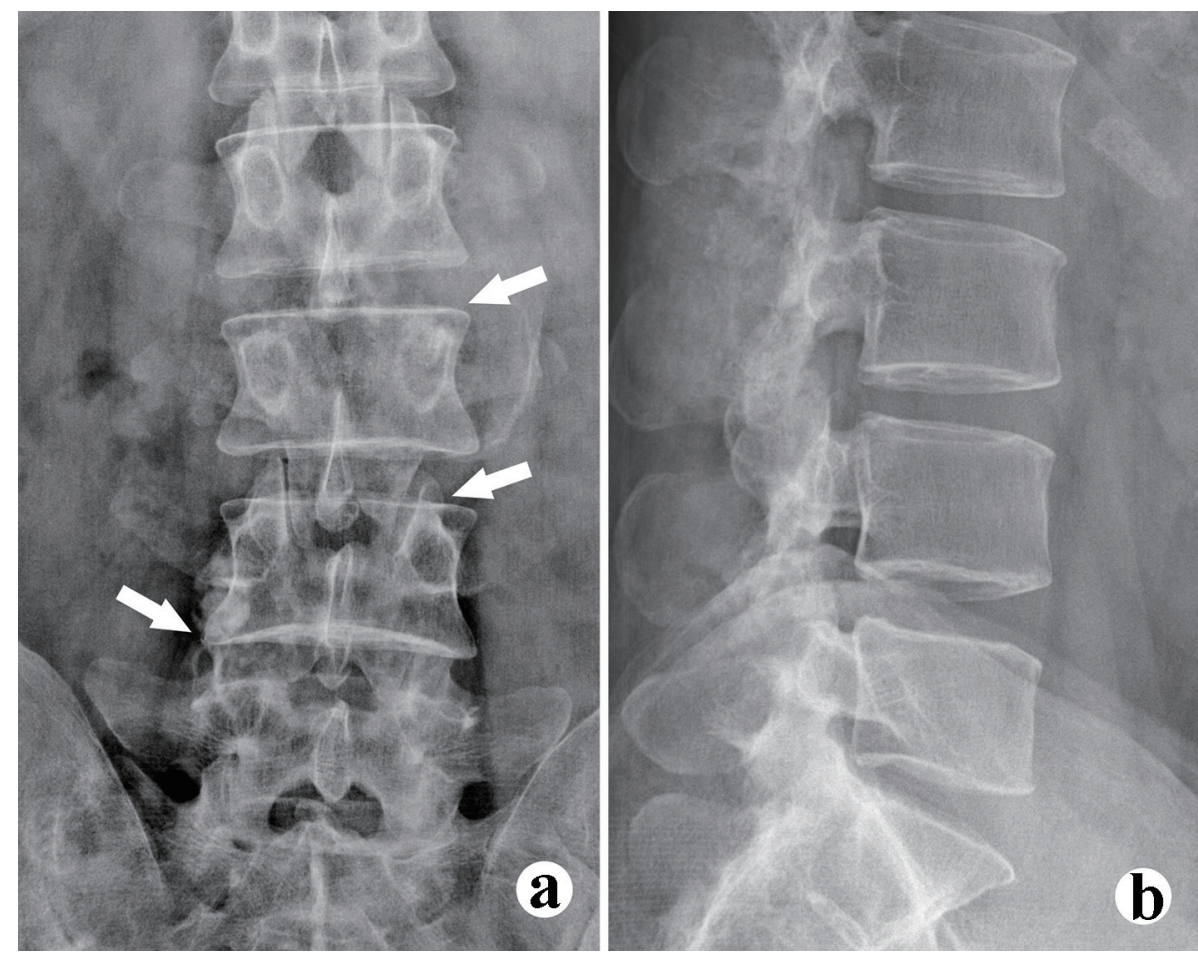

Figure 2. The X-ray films show the bony erosion and vague borderline of the tophi. (a) Anteroposterior (AP) view, facets erosion (arrow heads); (b) Lateral view. Vague borderline of the tophi can be observed. 

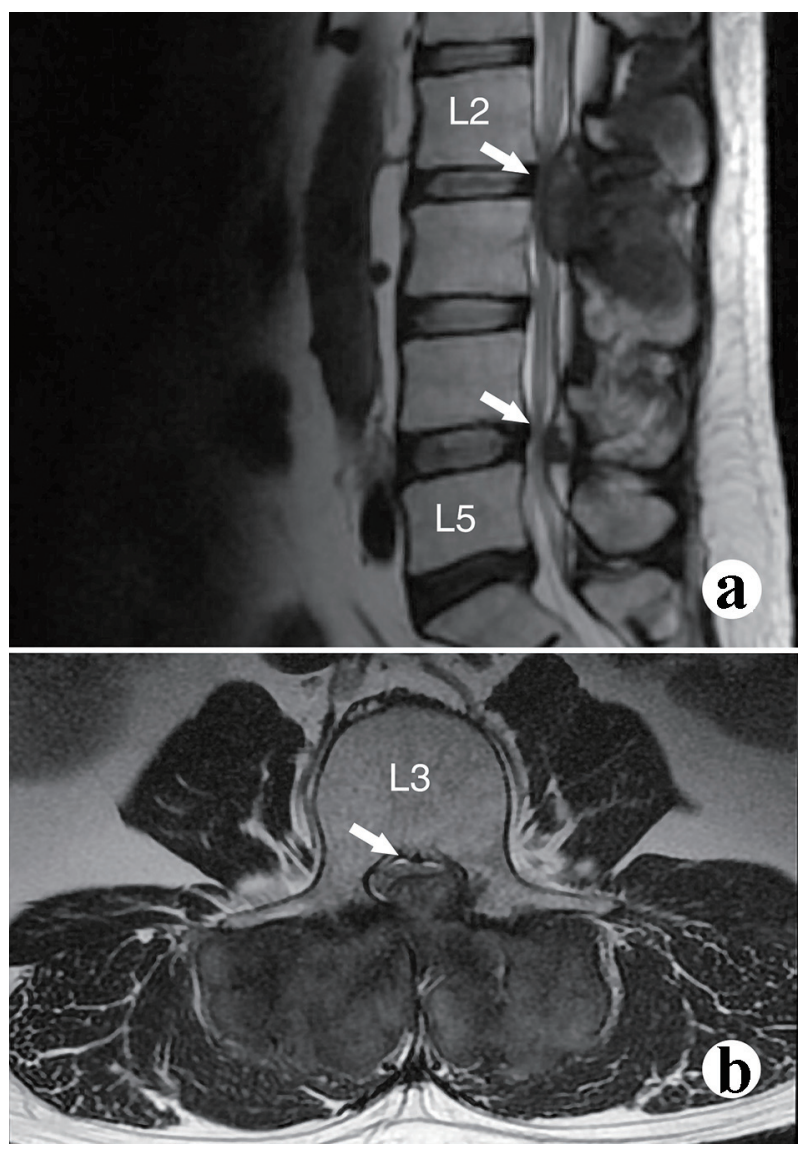

Figure 3. The magnetic resonance imaging (MRI) shows the severity of neurologic compression. (a) Mid-sagittal view, spinal stenosis at L2 and L4; (b) Axial view at upper L3 level. Severe spinal stenosis can be observed.

tive day 3. Steroids and Celebrex were used to deal with gout episodes that occurred postoperatively. Allopurinol, febuxostat and benzbromarone were adopted following the relief of gout episodes to reduce the serum UA level down to $478 \mu \mathrm{mol} / \mathrm{L}$. Postoperative histopathologic diagnosis confirmed the clinically gout tophi diagnosis (Fig. 5). The patient had a VAS score of 2, a JOA score of 25 and an ODI score of $5(10 \%)$ at 2 weeks and they turned out to be 1,27 and $3(6 \%)$ at 9 months postoperatively. The follow-up lumbar X-rays showed no recurrence of spinal tophi and no screw loosening.

\section{Discussion}

The elevation of serum uric acid (SUA) over a specific threshold will lead to the formation of monosodium urate (MSU) crystal, which can further induce acute gout attack under the stimulation of trauma, medication, alcohol drinking and drastic temperature change. The MSU is continuously deposited in the articular cartilage, synovium and connective tissue, which causes repeated inflammatory cell aggregation, epithelial cell wrapping and fibrous tissue proliferation to develop gout tophi. The tophus usually manifests about 10 years following the on-

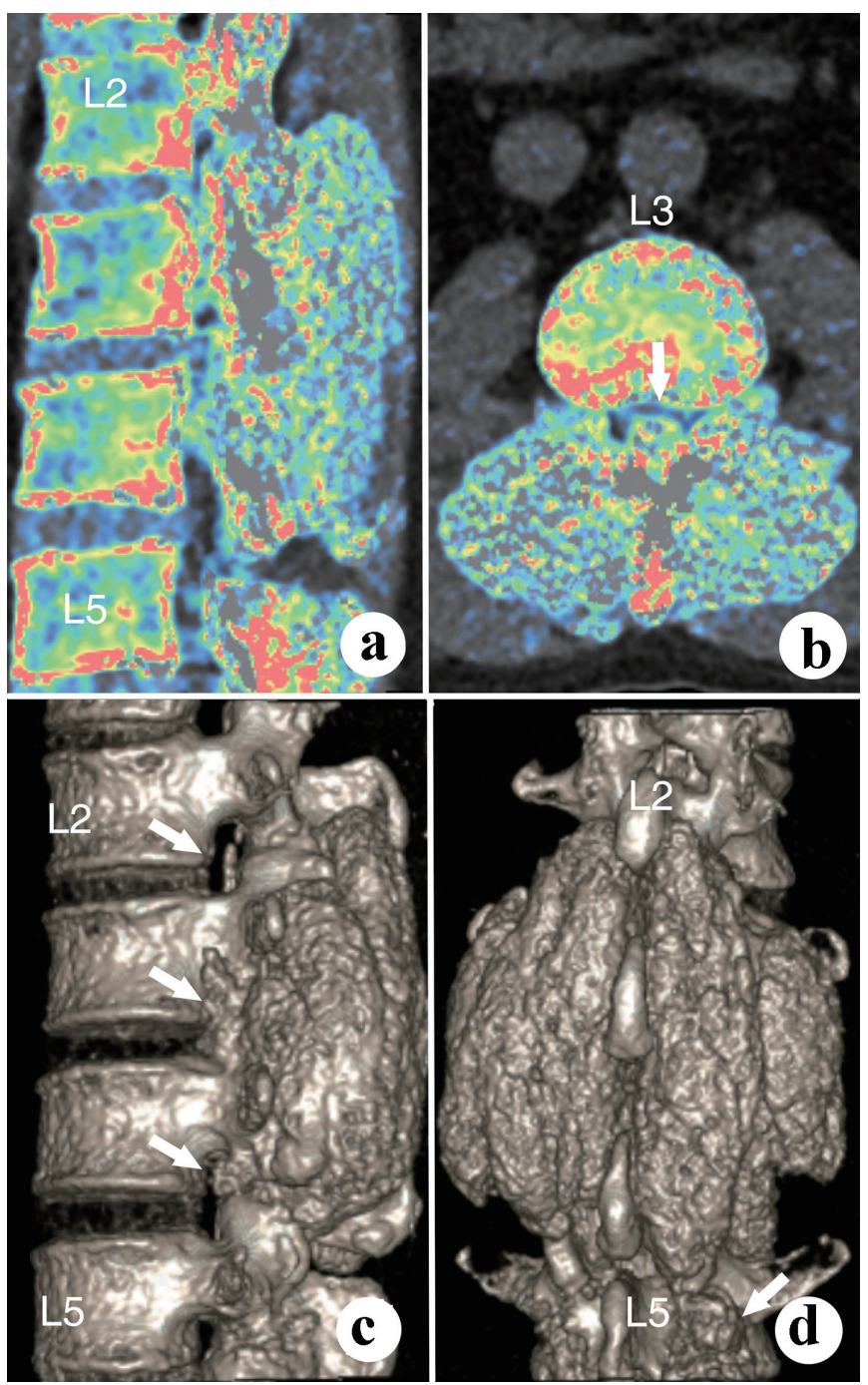

Figure 4. The dual-energy computed tomography shows the dimensions and volume of the tophi. (a) Para-sagittal view; (b) Axial view; (c) Lateral view; (d) Posterior view.

set of hyperuricemia. It is rare to see gout tophi that involve the spine, but it is even rarer to see spinal stenosis caused by a tophus encroachment into the spinal canal. To our knowledge, Koskoff et al [5] were the first to report spinal compression and paraplegia caused by a tophus. Afterwards, altogether 152 cases with spinal tophi were reported; therefore, clinical data for this condition remain inadequate. Hyperuricemia results from the increase of UA production and/or the decrease of UA elimination through the kidneys. According to the formula proposed by Boss et al [6], the hyperuricemia in this case was mainly caused by the decrease of elimination.

Tophus can affect almost all tissues including skin, joints, liver and other visceral organs with the first metatarsophalangeal joint and the auricles being the most commonly involved sites [7]. However, UA does not readily form tophi in the central nervous system due to the role of the blood-brain barrier. Although generally gout will not affect the spine, Toprover et al [8] suggest that spinal gout may be a common cause of axial 


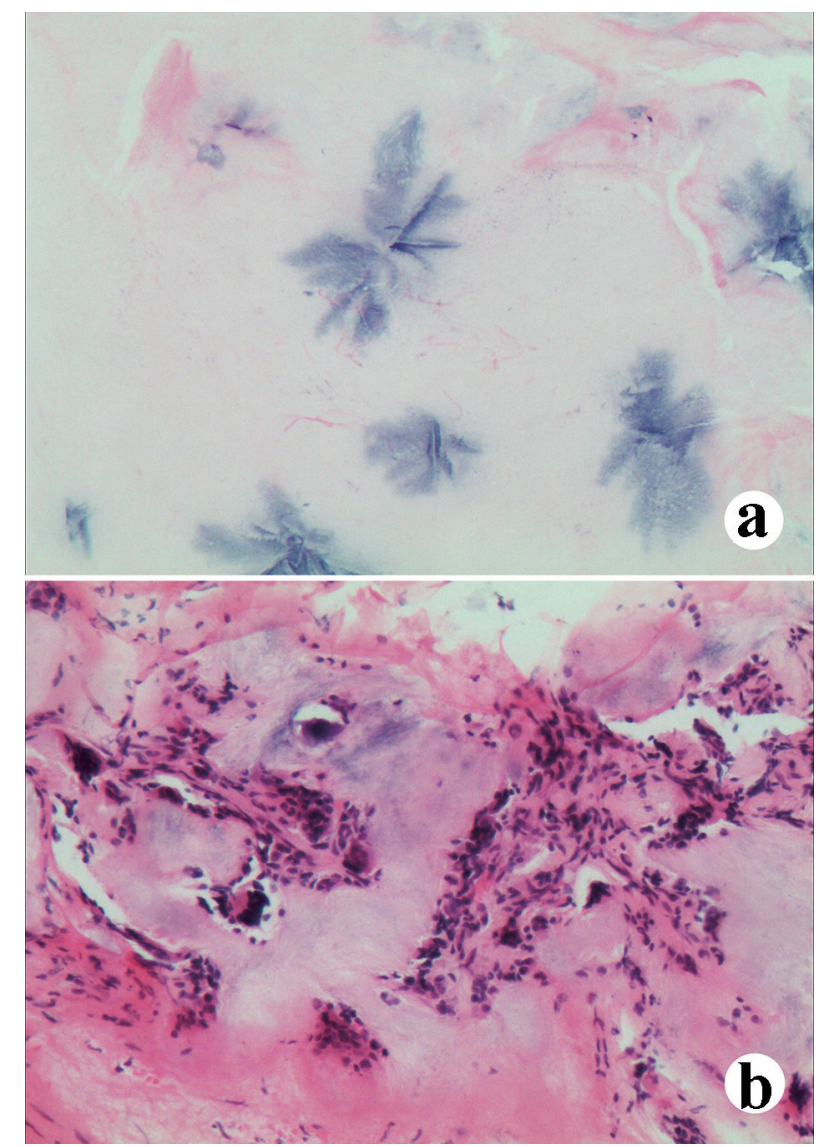

Figure 5. The pathological manifestations under microscope with hematoxylin and eosin (H\&E) staining. (a) Urate crystals can be observed (blue); (b) Inflammatory cell infiltration in the soft tissues invaded by the tophi.

pain that is not fully recognized. Gout can affect many areas of the spine, including vertebral bodies, pedicles, lamina, intervertebral discs, epidural space and even intradural spaces [9-11]. According to Sapsford et al [12], the MSU formed by $\mathrm{UA}$ and soft tissue inflammations leads to bony erosion. To our knowledge, the size of tophi in our case $(10 \times 10 \times 5 \mathrm{~cm})$ represents the largest amongst those reported in the current English literature.

Laboratory tests comprise the measurement of UA, creatinine, procalcitonin (PCT), CRP, ESR and WBC. The characteristic pathological change is birefringence MSU deposition under the polarized light microscope, which is the gold standard of gout tophus diagnosis. Imaging options include ultrasonography, X-ray, CT, MRI, positron emission tomography/ computed tomography and DECT.

Recently, the research and application on DECT has made significant progress on the diagnosis, treatment, follow-up and clinical research of spinal tophi. DECT has an advantage in the qualitative detection and overall evaluation of the gout source, and can detect gout deposited in atypical sites such as the spine $[13,14]$. A meta-analysis $[15]$ showed that the sensitivity and specificity of DECT were $87 \%$ and $84 \%$, respectively, compared with the pathological diagnosis of sodium urate crystal deposition as gold standard. The DECT of our case also supports the diagnosis of spinal gout tophi preoperatively.

The differential diagnoses of spinal tophi are spinal neoplasms, infection, ossification of posterior longitudinal ligament (OPLL), calcification of ligamentum flavum, calcium pyrophosphate dihydrate deposition (CPPD), etc. It is difficult to distinguish between gout and spinal infection when it causes pain and fever [16]. Tophi can cause spinal stenosis when they involve the spinal canal; however, MRI findings are difficult to distinguish them from spinal neoplasms when they are small [10]. In case of high UA, past tophus-attack history, or the existence of mucosa and joint tophi, and the patient also suffering from neck/low back pain, fever and even radiculopathy, the physicians should be alert to the possibility of spinal tophi, especially for those medical institutions without DECT.

Systemic and standardized medical treatment is necessary perioperatively to control acute inflammatory response, reduce UA formation and accelerate UA excretion. The commonly used medications include colchicine (acute phase), nonsteroidal antiinflammatory drugs (NSAIDS), steroids, febuxostat and benzbromarone (remission phase). Hemodialysis is required when the medications are ineffective. In 1953, the surgical treatment of paraplegia caused by thoracic tophus compression was reported for the first time [5]. Thereafter, surgery had become the mainstay to treat spinal tophi. Surgical decompression should be performed as soon as possible to reduce further soft tissue and bone invasion if the spinal tophi were diagnosed by biopsy and DECT, causing pain and spinal stenosis. Small spinal tophi can be removed under percutaneous endoscope, whereas open surgery is recommended for the complete removal of tophi involving multiple spinal segments [17]. Transforminal lumbar interbody fusion (TLIF) is feasible when there is spinal instability after decompression. There is often adhesion when a tophus involves the dura, thus we recommend that the decompression should be performed under microscope to reduce the risk of dural tear and neurologic injury. Some residues can be left if the adhesion is too tight, otherwise overzealous removal of tophi will increase the risk of dural tear, cerebrospinal fluid leakage and neurologic injury. Allogeneic blood transfusion might be needed when there is massive bleeding during multilevel spinal tophi excision procedures. Cortical screws (cortical bone trajectory screws) or vertebral body screws can be adopted to maintain spinal stability when there is severe pedicle erosion. It is generally not recommended to take the implants out unless the internal fixation fails. However, the internal fixation can be removed for younger patients with single-level fixation, no recurrence of tophi and good spinal stability after at least 1-year follow-up.

\section{Conclusion}

Despite the rare occurrence of spinal stenosis caused by tophi, for patients with obvious clinical manifestations of gout, especially at multiple sites, the clinicians should consider the possibility of spinal tophi. At present, surgery is the mainstay treatment, which should be tailored according to the extent of tophi erosion. DECT can acquire diagnosis before surgery and is meaningful for accurate preoperative evaluation and treat- 
ment. A lack of large case series were found in the current literature, therefore the comprehensive classification, diagnosis and treatment of spinal tophi should be further enhanced.

\section{Acknowledgments}

None to declare.

\section{Financial Disclosure}

This project was not supported by any grant or funding agency.

\section{Conflict of Interest}

None to declare.

\section{Informed Consent}

Informed consent was obtained from the patient for publication of this case report and images.

\section{Author Contributions}

Genlong Jiao contributed to conception and design of the study. Yang Liu contributed to acquisition of data and performed the study. Genlong Jiao, Yang Liu, Xueshi Li, Pan Zhou and Zhizhong Li contributed to drafting and revising the article.

\section{References}

1. Toprover M, Krasnokutsky S, Pillinger MH. Gout in the Spine: Imaging, Diagnosis, and Outcomes. Curr Rheumatol Rep. 2015;17(12):70.

2. Ribeiro da Cunha P, Peliz AJ, Barbosa M. Tophaceous gout of the lumbar spine mimicking a spinal meningioma. Eur Spine J. 2018;27(4):815-819.

3. Ding Y, Wang W, Jiang W, Zhang L, Wang T, Li Z. Tophaceous gout causing thoracic spinal cord compression: Case report and review of the literature. Neurochirurgie.
2018;64(3):171-176.

4. Zou Y, Li Y, Liu J, Zhang B, Gu R. Gouty spondylodiscitis with lumbar vertebral body retrolisthesis: A case report. Medicine (Baltimore). 2019;98(7):e14415.

5. Koskoff YD, Morris LE, Lubic LG. Paraplegia as a complication of gout. J Am Med Assoc. 1953;152(1):37-38.

6. Boss GR, Seegmiller JE. Hyperuricemia and gout. Classification, complications and management. N Engl J Med. 1979;300(26):1459-1468.

7. Ministrini S, Baronio G, Zorzi F, Bercich L, Grazioli L, Molfino S, Portolani N. Unusual presentation of gouty tophus in the liver with subsequent appearance in the same site of HCC: a correlate diagnosis? Case report. World J Surg Oncol. 2019;17(1):10.

8. Marinho F, Zeitoun-Eiss D, Renoux J, Brasseur JL, Genestie C, Grenier P. Tophaceous gout of the spine: case report and review of the literature. J Neuroradiol. 2012;39(2):123-126.

9. Wu D, Ma J, Li S, Zhao J, Li L. Spinal gout without spinal symptom in a junior school student: a case report. Spine (Phila Pa 1976). 2018;43(8):E488-E491.

10. Sapsford M, Gamble GD, Aati O, Knight J, Horne A, Doyle AJ, Dalbeth N. Relationship of bone erosion with the urate and soft tissue components of the tophus in gout: a dual energy computed tomography study. Rheumatology (Oxford). 2017;56(1):129-133.

11. Klauser AS, Halpern EJ, Strobl S, Abd Ellah MMH, Gruber J, Bellmann-Weiler R, Auer T, et al. Gout of hand and wrist: the value of US as compared with DECT. Eur Radiol. 2018;28(10):4174-4181.

12. Zielinska A, Nowakowska-Plaza A, Falkowski J, Stasiek M, Sudol-Szopinska I, Gluszko P. Dualenergy computed tomography: a novel imaging method in 2 cases of a rare course of gout. Pol Arch Intern Med. 2019;129(4):290291.

13. Ogdie A, Taylor WJ, Weatherall M, Fransen J, Jansen TL, Neogi T, Schumacher HR, et al. Imaging modalities for the classification of gout: systematic literature review and meta-analysis. Ann Rheum Dis. 2015;74(10):1868-1874.

14. Barrett K, Miller ML, Wilson JT. Tophaceous gout of the spine mimicking epidural infection: case report and review of the literature. Neurosurgery. 2001;48(5):11701172; discussion 1172-1173.

15. Xie L, Zhang X, Xi Z, Li J. Percutaneous endoscopic treatment for cervical ligamentum flavum gouty tophus: A case report. Medicine (Baltimore). 2019;98(20):e15665. 